\title{
ARITHMETICAL FUNCTIONS COMMUTABLE WITH SUMS OF SQUARES II.
}

\author{
Imre Kátai ${ }^{1, *}$ and Bui Minh Phong ${ }^{1, *}$
}

(Dedicated to Professor Ha Huy Khoai on occasion of his 75th birthday)

${ }^{1}$ ELTE, Pázmány P. Sétány. 1/C, H-1117 Budapest, Hungary

Communicated by László Tóth

Original Research Paper

Received: Aug 13, 2021 - Accepted: Sep 9, 2021

First published online: October 26, 2021

(c) 2021 The Author(s)

\section{ABSTRACT}

We give all functions $f, h: \mathbb{N} \rightarrow \mathbb{C}$ which satisfy the relation

$$
f\left(a^{2}+b^{2}+c^{2}+k\right)=h(a)+h(b)+h(c)+K
$$

for every $a, b, c \in \mathbb{N}$, where $k \geq 0$ is an integers and $K$ is a complex number. If $n$ cannot be written as $a^{2}+b^{2}+c^{2}+k$ for suitable $a, b, c \in \mathbb{N}$, then $f(n)$ is not determined. This is more complicated if we assume that $f$ and $h$ are multiplicative functions.

\section{KEYWORDS}

Arithmetical function, function equation, Dirichlet character, multiplicative function

\section{MATHEMATICS SUBJECT CLASSIFICATION (2020)}

Primary 11K65; Secondary 11N37, 11N64

\section{INTRODUCTION}

Let, as usual, $\mathcal{P}, \mathbb{N}_{0}, \mathbb{N}, \mathbb{Z}, \mathbb{C}$ be the set of primes, non negative integers, positive integers, integers and complex numbers, respectively. Let $\mathcal{M}$ be the set of all complex valued multiplicative functions.

We define $S$ and $\mathcal{A}$ as follows:

$$
S:=\left\{4^{s}(8 t+7) \mid s, t \in \mathbb{N}_{0}\right\}
$$

and

$$
\mathcal{A}:=\left\{r \in \mathbb{N} \mid r^{2}=k^{2}+h^{2}+t^{2} \text { and } r^{2}=u^{2}+v^{2} \text { for some } k, h, t, u, v \in \mathbb{N}\right\} .
$$

It is clear to check that

$$
\{7,15,23,28,31,39,47,55,60,63,71,79,87,92,95, \ldots\} \subseteq S
$$

and

$$
\{13,15,17,25,26,29,30,34,35,37,39,41,45,50, \ldots\} \subseteq \mathcal{A} \text {. }
$$

*Corresponding authors. E-mail: katai@inf.elte.hu and bui@inf.elte.hu 
For some $k \in \mathbb{N}$ let $\bar{k} \equiv k(\bmod 4)$ such that $\bar{k} \in\{0,1,2,3\}$. Let $\mathbb{N}_{k}=\{n \in \mathbb{N} \mid(n, k)=1\}$ and let $\mathcal{M}_{k}^{*}$ be the set of all completely multiplicative functions on the set $\mathbb{N}_{k}$.

Recently, in [8] and [9] we gave all arithmetical functions $f, h: \mathbb{N} \rightarrow \mathbb{C}$, which satisfy the relations

$$
f\left(a^{2}+b^{2}+c^{2}+d^{2}+k\right)=h(a)+h(b)+h(c)+h(d)+K
$$

for every $a, b, c, d \in \mathbb{N}$, where $k \in \mathbb{N}_{0}$ and $K \in \mathbb{C}$. We proved that (1.1) holds only if there are numbers $A, B \in \mathbb{C}$ such that

$$
h(m)=A m^{2}+B
$$

and

$$
f\left(a^{2}+b^{2}+c^{2}+d^{2}+k\right)=A\left(a^{2}+b^{2}+c^{2}+d^{2}\right)+4 B+K
$$

hold for every $n, m \in \mathbb{N}$.

Poo-Sung Park [6] proved that if $f \in \mathcal{M}$ and $k \in \mathbb{N}, k \geq 3$ satisfy one of following two conditions

$$
f\left(x_{1}^{2}+\cdots+x_{k}^{2}\right)=f\left(x_{1}\right)^{2}+\cdots+f\left(x_{k}\right)^{2}
$$

or

$$
f\left(x_{1}^{2}+\cdots+x_{k}^{2}\right)=f\left(x_{1}^{2}\right)+\cdots+f\left(x_{k}^{2}\right)
$$

for all positive integers $x_{1}, \ldots, x_{k}$, then $f$ is the identity function.

B.M.M.Khanh [4] determined the solution of the equation

$$
f\left(n^{2}+m^{2}+k\right)=f(n)^{2}+f(m)^{2}+K \quad(\forall n, m \in \mathbb{N}),
$$

where $k \in \mathbb{N}_{0}$ and $K \in \mathbb{C}$. In [3] and [5] she gave all functions $f: \mathbb{N} \rightarrow \mathbb{C}$ which satisfy the equation

$$
f\left(n^{2}+D m^{2}+k\right)=f(n)^{2}+D f(m)^{2}+k \text { for every } n, m \in \mathbb{N} .
$$

In this paper we improve the above results for two functions. We prove the following theorems. THEOREM 1.1. Let $k \in \mathbb{N}_{0}$ and $K \in \mathbb{C}$. Assume that the arithmetical functions $f, h: \mathbb{N} \rightarrow \mathbb{C}$ satisfy the equation

$$
f\left(a^{2}+b^{2}+c^{2}+k\right)=h(a)+h(b)+h(c)+K
$$

for every $a, b, c \in \mathbb{N}$. Then there are numbers $A, B, C \in \mathbb{C}$ such that

$$
h(m)=A m^{2}+B \chi_{2}(m)+C
$$

and

$$
f\left(a^{2}+b^{2}+c^{2}+k\right)=A\left(a^{2}+b^{2}+c^{2}\right)+B\left(\chi_{2}(a)+\chi_{2}(b)+\chi_{2}(c)\right)+D_{3},
$$

hold for every $m, a, b, c \in \mathbb{N}$, where $D_{3}=3 C+K$ and $\chi_{2}(r)$ is the Dirichlet character (mod 2), that is

$$
\chi_{2}(r)= \begin{cases}1 & \text { if }(r, 2)=1 \\ 0 & \text { if } 2 \mid r .\end{cases}
$$

Furthermore

$$
f\left(r^{2} n+k\right)=A r^{2} n+\bar{n} B \chi_{2}(r)+D_{3}
$$

holds for every $n \in \mathbb{N}, n \notin S$ and for every $r \in \mathcal{A}$.

In the following let

and for each $k \in \mathbb{N}_{0}$ let

$$
\mathbb{M}:=\left\{a^{2}+b^{2}+c^{2} \mid a, b, c \in \mathbb{N}\right\}
$$

$$
\mathcal{H}_{k}=\left\{\begin{array}{lll}
\{1, \ldots, e+2\} & \text { if } k=2^{e} k_{1},\left(k_{1}, 2\right)=1,2 \mid e \text { and } k_{1} \equiv 1 & (\bmod 8), \\
\mathbb{N} \backslash\{e+2\} & \text { if } k=2^{e} k_{1},\left(k_{1}, 2\right)=1,2 \mid e \text { and } k_{1} \equiv 5 & (\bmod 8), \\
\mathbb{N} & \text { in any other cases. }
\end{array}\right.
$$

THEOREM 1.2. Let $k \in \mathbb{N}_{0}$ and $K \in \mathbb{C}$. Assume that $F, H \in \mathcal{M}$ satisfy the relation

$$
F\left(a^{2}+b^{2}+c^{2}+k\right)=H(a)+H(b)+H(c)+K
$$

for every $a, b, c \in \mathbb{N}$.

Then one of the following assertions holds: 
(1) $H(m)=1$ and $F(\eta+k)=0(\forall m \in \mathbb{N}, \forall \eta \in \mathbb{M})$ if $K=-3$,

(2) $H(m)=1$ and $F(2 n+1)=1, F\left(2^{\alpha}\right)=K+3\left(\forall m, n \in \mathbb{N}, \forall \alpha \in \mathcal{H}_{k}\right)$ if $K \neq-3$,

(3) $H(m)=\chi_{2}(m)$ and $F(2 n+1)=1(\forall m, n \in \mathbb{N})$ if $(\bar{k}, K) \neq(3,-1)$,

(4) $H(m)=\chi_{2}(m)$ and $F(2 n+1)=(-1)^{n}, F(2)=(-1)^{\frac{k+1}{4}} 2, F\left(2^{\alpha}\right)=0\left(\forall m, n \in \mathbb{N}, \forall \alpha \in \mathcal{H}_{k}, \alpha \geq 2\right)$ if $(\bar{k}, K)=(3,-1)$,

(5) $H(m)=m^{2}, F(2 n+1)=2 n+1$ and $F\left(2^{\alpha}\right)=2^{\alpha}\left(\forall m, n \in \mathbb{N}, \forall \alpha \in \mathcal{H}_{k}\right)$.

We derive the following corollaries from Theorem 1.1.

COROLLARY 1.3. Let $\ell \in \mathbb{N}$ and $\ell \geq 3$. Assume that the arithmetical function $F: \mathbb{N} \rightarrow \mathbb{C}$ satisfies the relation

$$
F\left(n_{1}^{2}+\cdots+n_{\ell}^{2}\right)=F\left(n_{1}\right)^{2}+\cdots+F\left(n_{\ell}\right)^{2}
$$

for every $n_{1}, \ldots, n_{\ell} \in \mathbb{N}$. Then one of the following assertions holds:

(i) $F(n)=0$ for every $n \in \mathbb{N}$,

(ii) $F(n)=\frac{\varepsilon(n)}{\ell}$ for every $n \in \mathbb{N}$,

(iii) $F(n)=\varepsilon(n) n$ for every $n \in \mathbb{N}$, where $\varepsilon(n) \in\{-1,1\}$ and $\varepsilon\left(n_{1}^{2}+\cdots+n_{\ell}^{2}\right)=1$ for every $n_{1}, \ldots, n_{\ell} \in \mathbb{N}$.

COROLLARY 1.4. Let $\ell \in \mathbb{N}$ and $\ell \geq 4$. Assume that the arithmetical function $F: \mathbb{N} \rightarrow \mathbb{C}$ satisfies the relation

$$
F\left(n_{1}^{2}+\cdots+n_{\ell}^{2}\right)=F\left(n_{1}^{2}\right)+\cdots+F\left(n_{\ell}^{2}\right)
$$

holds for every $n_{1}, \ldots, n_{\ell} \in \mathbb{N}$. Then there is a complex $A$ such that

$$
F\left(m^{2}\right)=A m^{2} \text { for every } m \in \mathbb{N}
$$

and

$$
F\left(n_{1}^{2}+\cdots+n_{\ell}^{2}\right)=A\left(n_{1}^{2}+\cdots+n_{\ell}^{2}\right)
$$

hold for every $m, n_{1}, \ldots, n_{\ell} \in \mathbb{N}$. Consequently, $F(n)=A n$ for every $n$ which can be written as $n=n_{1}^{2}+\cdots+n_{\ell}^{2}$ for $n_{1}, \ldots, n_{\ell} \in \mathbb{N}$.

\section{PROOF OF THEOREM 1.1}

Assume that the arithmetical functions $f, h: \mathbb{N} \rightarrow \mathbb{C}$ satisfy the relation

$$
f\left(a^{2}+b^{2}+c^{2}+k\right)=h(a)+h(b)+h(c)+K
$$

for every $a, b, c \in \mathbb{N}$.

We shall prove that there are numbers $A, B, C \in \mathbb{C}$ such that

$$
h(m)=A m^{2}+B \chi_{2}(m)+C
$$

and

$$
f\left(a^{2}+b^{2}+c^{2}+k\right)=A\left(a^{2}+b^{2}+c^{2}\right)+B\left(\chi_{2}(a)+\chi_{2}(b)+\chi_{2}(c)\right)+D_{3},
$$

hold for every $m, a, b, c \in \mathbb{N}$, where $D_{3}=3 C+K$ and $\chi_{2}(r)$ is the principal character (mod 2).

We define numbers $A, B, C$ as follows:

$$
A=\frac{h(3)-h(1)}{8}, B=\frac{3 h(3)-8 h(2)+5 h(1)}{8} \text { and } C=\frac{-h(3)+2 h(2)+h(1)}{2} .
$$

It is obvious from the definitions of $A, B$ and $C$ that (2.2) is true for $m \in\{1,2,3\}$. Indeed, we have $A \cdot 1^{2}+B \chi_{2}(1)+C=A+B+C=\frac{h(3)-h(1)}{8}+\frac{3 h(3)-8 h(2)+5 h(1)}{8}+\frac{-h(3)+2 h(2)+h(1)}{2}=h(1)$, $A \cdot 2^{2}+B \chi_{2}(2)+C=4 A+C=4 \frac{h(3)-h(1)}{8}+\frac{-h(3)+2 h(2)+h(1)}{2}=h(2)$ and

$A \cdot 3^{2}+B \chi_{2}(3)+C=9 A+B+C=9 \frac{h(3)-h(1)}{8}+\frac{3 h(3)-8 h(2)+5 h(1)}{8}+\frac{-h(3)+2 h(2)+h(1)}{2}=h(3)$.

Since $5^{2}+1^{2}+1^{2}=3^{2}+3^{2}+3^{2}$, we infer from (2.1) that

$$
h(5)+2 h(1)+K=f\left(5^{2}+1^{2}+1^{2}+k\right)=f\left(3^{2}+3^{2}+3^{2}+k\right)=3 h(3)+K
$$


and so

$$
h(5)=3 h(3)-2 h(1) .
$$

Now we infer from the fact $5^{2}+2^{2}+2^{2}=4^{2}+4^{2}+1^{2}$ that $h(5)+2 h(2)+K=2 h(4)+h(1)+K$, which implies

$$
h(4)=\frac{h(5)+2 h(2)-h(1)}{2}=\frac{(3 h(3)-2 h(1))+2 h(2)-h(1)}{2}=\frac{3 h(3)+2 h(2)-3 h(1)}{2} .
$$

On the other hand, we have

$$
A \cdot 4^{2}+B \chi_{2}(4)+C=16 \frac{h(3)-h(1)}{8}+\frac{-h(3)+2 h(2)+h(1)}{2}=\frac{3 h(3)+2 h(2)-3 h(1)}{2},
$$

which proves that $h(4)=A \cdot 4^{2}+B \chi_{2}(4)+C$, and so (2.2) is true for $m=4$.

Assume that (2.2) is true for every $m<M$, where $M \geq 5$. Since $M-4 \in \mathbb{N}$ and $M-2 \in \mathbb{N}$, we infer from the next relation

$$
M^{2}+(M-4)^{2}+1^{2}=(M-2)^{2}+(M-2)^{2}+3^{2},
$$

that

$$
h(M)+h(M-4)+h(1)=2 h(M-2)+h(3) .
$$

Thus, from our assumptions, we have

$$
\begin{aligned}
h(M) & =2 h(M-2)-h(M-4)+h(3)-h(1) \\
& =2\left(A(M-2)^{2}+B \chi_{2}(M-2)+C\right)-\left(A(M-4)^{2}+B \chi_{2}(M-4)+C\right)+h(3)-h(1) \\
& =A M^{2}+B \chi_{2}(M)+C,
\end{aligned}
$$

which proves that (2.2) holds for $M$, and so (2.2) holds for every $m \in \mathbb{N}$.

Finally, from (2.1) and (2.2), we have

$$
\begin{aligned}
f\left(a^{2}+b^{2}+c^{2}+k\right) & =h(a)+h(b)+h(c)+K \\
& =\left(A a^{2}+B \chi_{2}(a)+C\right)+\left(A b^{2}+B \chi_{2}(b)+C\right)+\left(A c^{2}+B \chi_{2}(c)+C\right)+K \\
& =A\left(a^{2}+b^{2}+c^{2}\right)+B\left(\chi_{2}(a)+\chi_{2}(b)+\chi_{2}(c)\right)+3 C+K .
\end{aligned}
$$

The proof of (2.3) is finished with $D_{3}=3 C+K$.

Now we prove that

$$
f\left(r^{2} n+k\right)=A r^{2} n+\bar{n} B \chi_{2}(r)+D_{3}
$$

holds for every $n \in \mathbb{N}, n \notin S$ and for every $r \in \mathcal{A}$, where $\bar{n} \in\{0,1,2,3\}$ such that $n \equiv \bar{n}(\bmod 4)$.

In order to prove (2.4), we need the following result concerning the sum of three squares.

LEMMA 2.1 (Legendre's three-square theorem). A number $n \in \mathbb{N}$ can be represented as the sum of three squares of integers if and only if $n \notin S$.

Let $n \in \mathbb{N}, n \notin \mathcal{S}, r \in \mathcal{A}, r^{2}=k^{2}+h^{2}+t^{2}$ and $r^{2}=u^{2}+v^{2}$ for some $k, h, t, u, v \in \mathbb{N}$. Since $n \notin \mathcal{S}$, Lemma 2.1 implies that $n=x^{2}+y^{2}+z^{2}$ with $x, y, z \in \mathbb{Z}$. Then there are three options:

(a) $n=x^{2}+y^{2}+z^{2}$ with $x, y, z \in \mathbb{N}$,

(b) $n=x^{2}+y^{2}$ with $x, y \in \mathbb{N}$,

(c) $n=x^{2}$ with $x \in \mathbb{N}$.

In the case (a), we have $r^{2} n=(r x)^{2}+(r y)^{2}+(r z)^{2}$. It is clear to check that

$$
\chi_{2}(r x)+\chi_{2}(r y)+\chi_{2}(r z)=\bar{n} \chi_{2}(r) .
$$

Indeed, since $n=x^{2}+y^{2}+z^{2}$, we have

$$
(\bar{n}, \bar{x}, \bar{y}, \bar{z}) \in\{(0,0,0,0),(1,1,0,0),(2,1,1,0),(3,1,1,1)\},
$$

which proves (2.5). Therefore (2.3) and (2.5) imply

$$
\begin{aligned}
f\left(r^{2} n+k\right) & =f\left((r x)^{2}+(r y)^{2}+(r z)^{2}+k\right) \\
& =A\left((r x)^{2}+(r y)^{2}+(r z)^{2}\right)+B\left(\chi_{2}(r x)+\chi_{2}(r y)+\chi_{2}(r z)\right)+D_{3} \\
& =A r^{2} n+\bar{n} B \chi_{2}(r)+D_{3},
\end{aligned}
$$


which proves (2.4) for the case (a).

In the case (b), we have $r^{2} n=(r x)^{2}+(r y)^{2}=(r x)^{2}+(u y)^{2}+(v y)^{2}$ and

$$
\chi_{2}(r x)+\chi_{2}(u y)+\chi_{2}(v y)=\bar{n} \chi_{2}(r) \text {. }
$$

This with (2.3) implies (2.4).

In the case (c), we have

$$
r^{2} n=\left(k^{2}+h^{2}+t^{2}\right) x^{2}=(k x)^{2}+(h x)^{2}+(t x)^{2}
$$

and

$$
\chi_{2}(k x)+\chi_{2}(h x)+\chi_{2}(t x)=\bar{n} \chi_{2}(r)
$$

which by using (2.3) proves (2.4). Therefore, the proof of (2.4) is finished.

Theorem 1.1 is proved.

\section{LEMMAS FOR THE PROOF OF THEOREM 1.2}

LEMMA 3.1 (P. D. T. A. Elliott, [1] Lemma 19.3). Assume that $F \in \mathcal{M}$ satisfies the relation

$$
F(a n+b)=d
$$

for every $n \in \mathbb{N}$, where $a, b \in \mathbb{N}$ and $d \in \mathbb{C} \backslash\{0\}$. Then $F(n)=\chi_{a}(n)$ for every $n \in \mathbb{N}_{a}$, where $\chi_{a}(n)$ is a Dirichlet real character $(\bmod a)$.

Proof. This 1emma is identical to Lemma 19.3 in [1].

LEMMA 3.2. Assume that $F \in \mathcal{M}$ satisfies the equation

$$
F(a n+b)=c n+d
$$

for every $n \in \mathbb{N}$, where $a, b \in \mathbb{N}$ and $c, d \in \mathbb{C}$ are given. Then one of the following assertions holds:

(a) $c=d=0$ and $F(a n+b)=0$ for every $n \in \mathbb{N}$,

(b) $c=0, F(b)=d \neq 0$ and $F(n)=\chi_{a}(n)$ for every $n \in \mathbb{N}_{a}$,

(c) $c \neq 0, a d=b c, F(b)=d \neq 0$ and $F(n)=\chi_{a}(n) n$ for every $n \in \mathbb{N}_{a}$,

where $\chi_{a}(n)$ is a Dirichlet real character $(\bmod a)$.

Proof. Let $M \in \mathbb{N}, M \equiv 1(\bmod a)$ be an arbitrary. Let

$$
I_{M}:=\{n \in \mathbb{N} \mid(M, a n+b)=1 .\}
$$

It follows from the fact $M \equiv 1(\bmod a)$ that $\left|I_{M}\right|=\infty$. We infer from the facts $F \in \mathcal{M}$ and (3.1) that

$$
\begin{aligned}
c F(M) n+d F(M) & =F(M)(c n+d)=F(M) F(a n+b)= \\
& =F\left[a\left(M n+\frac{M-1}{a} b\right)+b\right]=c\left(M n+\frac{M-1}{a} b\right)+d= \\
& =c M n+b c \frac{M-1}{a}+d
\end{aligned}
$$

holds for every $n \in I_{M}$, consequently

$$
\left\{\begin{array}{l}
c F(M)=c M \\
d F(M)=b c \frac{M-1}{a}+d .
\end{array}\right.
$$

The first equation shows that either $c=0$ or $F(M)=M$. If $c=0$, then the second equation gives $d F(M)=d$, consequently either $d=0$ or $F(M)=1$. If $(c, d)=(0,0)$, then $F(a n+b)=0$ for every $n \in \mathbb{N}$, and so (a) is proved.

If $c=0, d \neq 0$, then the second equation shows that $F(M)=1$ holds for every $M \in \mathbb{N}, M \equiv 1$ $(\bmod a)$. Lemma 3.1 implies that $F(n)=\chi_{a}(n)$ for every $n \in \mathbb{N}_{a}$. Since $F \in \mathcal{M}_{a}^{*}$ and $F(n)=\chi_{a}(n)$ for every $n \in \mathbb{N}_{a}$, we infer from (3.1) that

$$
F(b)=F(b) F(a t+1)=F(a b t+b)=d,
$$

which proves (b) of Lemma 3.2 . 
Now assume that $c \neq 0$ and $F(M)=M$ for every $M \in \mathbb{N}, M \equiv 1(\bmod a)$. Let $g(n):=\frac{F(n)}{n}$ for every $n \in \mathbb{N}$. Then $g \in \mathcal{M}$ and $g(M)=1$ for every $M \in \mathbb{N}, M \equiv 1(\bmod a)$, Lemma 3.1 implies that $g(n)=\chi_{a}(n)$ for every $n \in \mathbb{N}_{a}$. Therefore

$$
F(n)=\chi_{a}(n) n \text { for every } n \in \mathbb{N}_{a} .
$$

Now we prove that

$$
a d=b c \text { and } F(b)=d .
$$

Indeed, since $F \in \mathcal{M}_{a}^{*}$ and $F(n)=\chi_{a}(n) n$ for every $n \in \mathbb{N}_{a}$, we infer from (3.1) that

$$
F(b)(a t+1)=F(b) F(a t+1)=F(a b t+b)=b c t+d,
$$

which implies that

$$
a F(b)=b c, F(b)=d \text { and } a d=b c .
$$

Since $c \neq 0, b \in \mathbb{N}$, we have $d \neq 0$, and so the above relation proves the assertion (c) of Lemma 3.2. Lemma 3.2 is proved.

LEMMA 3.3. Let $a, b \in \mathbb{N}, D=(a, b)$. If $F \in \mathcal{M}$ such that $F(n)=\chi_{a}(n)$ for every $n \in \mathbb{N}_{a}$ and $F(n)=\chi_{b}(n)$ for every $n \in \mathbb{N}_{b}$, then $F(n)=\chi_{D}(n)$ for every $n \in \mathbb{N}_{D}$.

Proof. Since $F$ is a completely multiplicative function on $\mathbb{N}_{a}$ and $\mathbb{N}_{b}$, we have $F$ is completely multiplicative function on $\mathbb{N}_{D}$. We prove that $F$ is periodic $(\bmod D)$.

Since $F(n)=\chi_{a}(n)$ for every $n \in \mathbb{N}_{a}$ and $F(n)=\chi_{b}(n)$ for every $n \in \mathbb{N}_{b}$, we have $F$ is periodic $(\bmod a)$ and $(\bmod b)$. Let $u, v \in \mathbb{N}$ such that $D=(a, b)=a u-b v$. Then

$$
F(n+D)=F(n+D+b v)=F(n+a u)=F(n) \text { for every } n \in \mathbb{N}_{D} .
$$

Therefore, we have $F(N)=\chi_{D}(n)$. Lemma 3.3 is proved.

LEMMA 3.4. Let $k \in \mathbb{N}_{0}$ and

$$
\mathcal{H}_{k}:=\left\{\alpha \in \mathbb{N} \mid 2^{\alpha} \| a^{2}+b^{2}+c^{2}+k \text { for some } a, b, c \in \mathbb{N} .\right\}
$$

Then

$$
\mathcal{H}_{k}=\left\{\begin{array}{lll}
\{1, \ldots, e+2\} & \text { if } k=2^{e} k_{1},\left(k_{1}, 2\right)=1,2 \mid e \text { and } k_{1} \equiv 1 & (\bmod 8), \\
\mathbb{N} \backslash\{e+2\} & \text { if } k=2^{e} k_{1},\left(k_{1}, 2\right)=1,2 \mid e \text { and } k_{1} \equiv 5 & (\bmod 8), \\
\mathbb{N} & \text { in any other cases. }
\end{array}\right.
$$

Proof. First we consider the case $k=0$. Since

$$
2^{2 j} \| 2^{2 j}+2^{2 j+2}+2^{2 j+2} \text { and } 2^{2 j+1} \| 2^{2 j}+2^{2 j}+2^{2 j+2},
$$

consequently $2 j \in \mathcal{H}_{0}$ and $2 j+1 \in \mathcal{H}_{0}$ for every $j \in \mathbb{N}$. This shows that $\mathcal{H}_{0}=\mathbb{N}$.

Let $k>0, k=2^{e} k_{1},\left(k_{1}, 2\right)=1$ and

$$
\mathbb{M}_{0}:=\left\{a^{2}+b^{2}+c^{2} \mid a, b, c \in \mathbb{N}_{0}\right\} \text { and } \mathbb{M}:=\left\{a^{2}+b^{2}+c^{2} \mid a, b, c \in \mathbb{N}\right\} .
$$

It is known that $n \in \mathbb{M}_{0}$ if and only if $n \notin S$ and if $\eta=a^{2}+b^{2}+c^{2} \in \mathbb{M}_{0}$ with $a, b, c \in \mathbb{N}_{0}$, then

$$
\eta_{t}=\left(a+2^{t}\right)^{2}+\left(b+2^{t}\right)^{2}+\left(c+2^{t}\right)^{2} \in \mathbb{M} \text { and } \eta_{t}-\eta \equiv 0 \quad\left(\bmod 2^{t+1}\right)
$$

hold for every $t \in \mathbb{N}$. Therefore, if $\alpha \in \mathbb{N}$ such that $2^{\alpha} \| \eta+k$ for some $\eta \in \mathbb{M}_{0}$, then $2^{\alpha} \| \eta_{t}+k$ for every $t \in \mathbb{N}, t \geq \alpha$. This means that

$$
\text { If } 2^{\alpha} \| \eta+k \text { for some } \eta \in \mathbb{M}_{0} \text {, then } \alpha \in \mathcal{H}_{k} \text {. }
$$

Since $k=2^{e} k_{1},\left(k_{1}, 2\right)=1$, we consider the equation

$$
\eta+2^{e} k_{1}=2^{\alpha} r,(r, 2)=1 \text {, where } \eta \in \mathbb{M}_{0}, \alpha, r \in \mathbb{N} \text { are unknown. }
$$

CASE 1. $2 \mid e$

CASE 1a. $2 \mid e, \alpha \geq e$. 
If (3.2) is true, then $2^{e} \mid \eta$, and so

$$
\theta=\frac{\eta}{e^{e}} \in \mathbb{M}_{0} \Longleftrightarrow \eta \in \mathbb{M}_{0}
$$

thus (3.2) is true if and only if

$$
\theta+k_{1}=2^{\alpha-e} r,(r, 2)=1, \theta \in \mathbb{M}_{0}, \alpha, r \in \mathbb{N} .
$$

- If $\alpha \geq e+3$, then

$$
\theta \notin \mathbb{M}_{0} \Longleftrightarrow \theta=2^{\alpha-e} r-k_{1} \in S \Longleftrightarrow k_{1} \equiv 1 \quad(\bmod 8),
$$

and so no exist solution $\theta \in \mathbb{M}_{0}$ if $\alpha \geq e+3$ and $k_{1} \equiv 1(\bmod 8)$ and in any other case (3.3) has a solution.

$\circ$ If $\alpha=e+2$, then

$$
\theta \notin \mathbb{M}_{0} \Longleftrightarrow \theta=2^{2} r-k_{1} \in S \Longleftrightarrow k_{1} \equiv 5 \quad(\bmod 8) .
$$

Therefore there is no solution $\theta \in \mathbb{M}_{0}$ if $\alpha=e+2$ and $k_{1} \equiv 5(\bmod 8)$ and in any other case (3.3) has a solution.

-If $\alpha=e+1$, then $\eta=\overline{k_{1}} 2^{e} \in \mathbb{M}_{0}$ is a solution of (3.2).

- If $\alpha=e$, then $\eta=2^{e}+2^{e} \in \mathbb{M}_{0}$ is a solution of (3.2).

CASE 1b. $2 \mid e, \alpha<e$.

Then $\eta=2^{e} \in \mathbb{M}_{0}$ is a solution of (3.2).

CASE 2. $2 \nmid e$.

First we prove that $\{1, \ldots, e\} \subset \mathcal{H}_{k}$. It is clear that $2 \| \eta_{1}+2^{e} k_{1}$ with $\eta_{1}$ is defined by

Let

$$
\eta_{1}= \begin{cases}2=1^{2}+1^{2} \in \mathbb{M}_{0} & \text { if } e>1 \\ 4=2^{2} \in \mathbb{M}_{0} & \text { if } e=1\end{cases}
$$

$$
\eta_{2}= \begin{cases}2^{2} \in \mathbb{M}_{0} & \text { if } e>2 \\ 2^{4} \in \mathbb{M}_{0} & \text { if } e=2 \\ \left(k_{1}-1\right)^{2}+1^{2}+1^{2} \in \mathbb{M}_{0} & \text { if } e=1 .\end{cases}
$$

Then $2^{2} \| \eta_{2}+2^{e} k_{1}$, because in the case $e=1$, we have

$$
\eta_{2}+2^{e} k_{1}=\left(k_{1}-1\right)^{2}+1^{2}+1^{2}+2 k_{1}=4\left(\left(\frac{k_{1}-1}{2}\right)^{2}+\frac{k_{1}-1}{2}+1\right) .
$$

Thus we have proved that $1 \in \mathcal{H}_{k}$ and $2 \in \mathcal{H}_{k}$.

Now for each $2 j<e$ and $2 \ell+1<e$, we have $2^{2 j} \in \mathbb{M}_{0}, 2^{2 \ell}+2^{2 \ell} \in \mathbb{M}_{0}$ and

$$
2^{2 j}\left\|2^{2 j}+2^{e} k_{1}, 2^{2 \ell+1}\right\| 2^{2 \ell}+2^{2 \ell}+2^{e} k_{1} \text { and } 2^{e} \| 2^{e+1}+2^{e+1}+2^{e+1}+2^{e} k_{1} .
$$

Thus, we proved that $\{1, \ldots, e\} \subseteq \mathcal{H}_{k}$.

Finally, for every $\alpha \geq e+1$, we infer from $2 \nmid e$ that $2^{\alpha} r-2^{e} k_{1}=2^{e}\left(2^{\alpha-e} r-k_{1}\right) \notin \mathcal{S}$, i.e $\eta=2^{\alpha} r-2^{e} k_{1} \in \mathbb{M}_{0}$. This shows that $\alpha \in \mathcal{H}_{k}$ for every $\alpha \geq e+1$, which with the fact $\{1, \ldots, e\} \subseteq \mathcal{H}_{k}$ shows that $\mathcal{H}_{k}=\mathbb{N}$.

The proof of Lemma 3.4 is finished.

\section{PROOF OF THEOREM 1.2}

Assume that $k \in \mathbb{N}_{0}, K \in \mathbb{C}$ and $F, H \in \mathcal{M}$ satisfy the relation (2.1) for every $a, b, c \in \mathbb{N}$. Then Theorem 1.1 shows that there are numbers $A, B, C, D_{3}=3 C+K \in \mathbb{C}$ such that (2.2) and (2.3) are satisfied for every $m, a, b, c \in \mathbb{N}$. Furthermore (2.2)-(2.4) are true, i.e.

$$
\begin{gathered}
H(m)=A m^{2}+B \chi_{2}(m)+C, \\
F\left(a^{2}+b^{2}+c^{2}+k\right)=A\left(a^{2}+b^{2}+c^{2}\right)+B\left(\chi_{2}(a)+\chi_{2}(b)+\chi_{2}(c)\right)+D_{3},
\end{gathered}
$$


and

$$
F\left(r^{2} n+k\right)=A r^{2} n+\bar{n} B \chi_{2}(r)+D_{3}
$$

hold for every $m, n \in \mathbb{N}, n \notin S$ and $r \in \mathcal{A}$, where $\chi_{2}(r)$ is the principal character $(\bmod 2)$ and $D_{3}=3 C+K$.

We shall deduce from (4.2) and the fact $H \in \mathcal{M}$ that only the following three cases can occur:

$$
\left\{\begin{array}{lll}
(1) \quad(A, B, C)=(0,0,1) \text { and } H(m)=1 & \text { for every } m \in \mathbb{N}, \\
(2) \quad(A, B, C)=(0,1,0) \text { and } H(m)=\chi_{2}(m) & \text { for every } m \in \mathbb{N}, \\
(3) \quad(A, B, C)=(1,0,0) \text { and } H(m)=m^{2} & \text { for every } m \in \mathbb{N} .
\end{array}\right.
$$

Indeed, since $H \in \mathcal{M}$, we have

$$
\begin{aligned}
0 & =H(n m)-H(n) H(m)= \\
& =A n^{2} m^{2}+B \chi_{2}(n m)+C-\left(A n^{2}+B \chi_{2}(n)+C\right)\left(A m^{2}+B \chi_{2}(m)+C\right)= \\
& =\left(A-A^{2}\right) n^{2} m^{2}-A n^{2}\left(B \chi_{2}(m)+C\right)-A m^{2}\left(B \chi_{2}(n)+C\right)+B \chi_{2}(n m)+C-\left(B \chi_{2}(n)+C\right)\left(B \chi_{2}(m)+C\right)
\end{aligned}
$$

for every $n, m \in \mathbb{N},(n, m)=1$. This implies that

$$
\left\{\begin{aligned}
A & =A^{2}, B=B^{2} \text { and } C=C^{2} \\
A B & =A C=B C=0
\end{aligned}\right.
$$

Since $H(1)=A+B+C=1$, these relations imply that

$$
(A, B, C) \in\{(0,0,1),(0,1,0),(1,0,0)\}
$$

which proves (4.4).

In the proof of Theorem 1.2 we distinguish the cases 1, 2, 3 in (4.4).

(1) Assume that $(A, B, C)=(0,0,1)$ and $H(m)=1$ for every $m \in \mathbb{N}$. Then we infer from (4.2) and (4.3) that

$$
F(\eta+k)=D_{3}
$$

hold for every $\eta \in \mathbb{M}$, where $D_{3}=3 C+K=3+K$.

Since $4 n+1 \notin S$ and $4 n+2 \notin S$ for every $n \in \mathbb{N}$, we infer from (4.3) that

$$
F\left(4 r^{2} n+r^{2}+k\right)=D_{3} \text { and } F\left(4 r^{2} n+2 r^{2}+k\right)=D_{3}
$$

is satisfied for every $n \in \mathbb{N}$ and $r \in \mathcal{A}$.

- If $D_{3}=0, K=-3$, then $H(m)=C=1$ and $F(\eta+k)=0$ for every $m \in \mathbb{N}, \eta \in \mathbb{M}$, which proves (1) of Theorem 1.2.

- If $D_{3} \neq 0$, then it follows from (4.5) that

$$
\begin{cases}F(2) F\left(2 r^{2} n+r^{2}+\frac{k}{2}\right)=D_{3} & \text { if } \bar{k}=0,(r, 2)=1, \\ F(2) F\left(2 r^{2} n+\frac{r^{2}+k}{2}\right)=D_{3} & \text { if } \bar{k}=1,(r, 2)=1, \\ F\left(2^{\alpha_{r}}\right) F\left(2 r^{2} n+\frac{2 r^{2}+k}{2^{\alpha_{r}}}\right)=D_{3} & \text { if } \bar{k}=2,(r, 2)=1, \\ F\left(2^{\beta_{r}}\right) F\left(2 r^{2} n+\frac{r^{2}+k}{2^{\beta_{r}}}\right)=D_{3} & \text { if } \bar{k}=3,(r, 2)=1,\end{cases}
$$

where $2^{\alpha_{r}}\left\|2 r^{2}+k, 2^{\beta_{r}}\right\| r^{2}+k,\left(\alpha_{2} \geq 2, \beta_{r} \geq 2\right)$. These with Lemma 3.1 imply that

$$
F(n)=\chi_{2 r^{2}}(n) \text { for every } n \in \mathbb{N}_{2 r^{2}}, r \in \mathcal{A},(r, 2)=1,
$$

which with $r=13$ and $r=15$ shows that

$$
F(n)=\chi_{4 \cdot 13^{2}}(n) \text { and } F(n)=\chi_{4 \cdot 15^{2}}(n) .
$$

Therefore Lemma 3.3 implies that

$$
F(n)=\chi_{\left(2 \cdot 13^{2}, 2 \cdot 15^{2}\right)}(n)=\chi_{2}(n)=1 \text { for every } n \in \mathbb{N}_{2},
$$

which implies that $F(2 n+1)=1$ for every $m \in \mathbb{N}$. Thus, we infer from Lemma 3.4 and the fact $F(\eta+k)=D_{3}$ that $F\left(2^{\alpha}\right)=D_{3}$ for every $\alpha \in \mathcal{H}_{k}$. The assertion (2) of Theorem 1.2 is proved. 
(2) Assume now that $(A, B, C)=(0,1,0)$ and $H(m)=\chi_{2}(m)$ for every $m \in \mathbb{N}$. Then $D_{3}=3 C+K=K$ and

$$
F\left(a^{2}+b^{2}+c^{2}+k\right)=\chi_{2}(a)+\chi_{2}(b)+\chi_{2}(c)+K
$$

hold for every $a, b, c \in \mathbb{N}$, furthermore

$$
F\left(4 r^{2} n+r^{2}+k\right)=\chi_{2}(r)+K \text { and } F\left(4 r^{2} n+2 r^{2}+k\right)=2 \chi_{2}(r)+K
$$

is satisfied for every $n \in \mathbb{N}$ and $r \in \mathcal{A}$. If $r$ is odd, then at least one of $K+\chi_{2}(r)=K+1$, or $K+2 \chi_{2}(r)=K+2$ is not zero. Let $K_{1} \in\{K+1, K+2\}$ such that $K_{1} \neq 0$. Thus, it follows from (4.7) and Lemma 3.1 that

$$
F(n)=\chi_{4 r^{2}}(n) \text { for every } n \in \mathbb{N}_{4 r^{2}} \text { and } r \in \mathcal{A},(r, 2)=1 .
$$

Since this relation holds for every $r \in \mathcal{A},(r, 2)=1$, then an application of Lemma 3.3 with $r=13$ and $r=15$, we have

$$
F(n)=\chi_{\left(4 \cdot 13^{2}, 4 \cdot 15^{2}\right)}(n)=\chi_{4}(n) \text { for every } n \in \mathbb{N}_{4} .
$$

On the other hand, it follows from (4.7) that

$$
\begin{cases}F(2) F\left(2 r^{2} n+r^{2}+\frac{k}{2}\right)=2+K & \text { if } \bar{k}=0,(r, 2)=1, \\ F(2) F\left(2 r^{2} n+\frac{r^{2}+k}{2}\right)=1+K & \text { if } \bar{k}=1,(r, 2)=1, \\ F\left(2^{\alpha_{r}}\right) F\left(2 r^{2} n+\frac{2 r^{2}+k}{2^{\alpha_{r}}}\right)=2+K & \text { if } \bar{k}=2,(r, 2)=1, \\ F\left(2^{\beta_{r}}\right) F\left(2 r^{2} n+\frac{r^{2}+k}{2^{\beta_{r}}}\right)=1+K & \text { if } \bar{k}=3,(r, 2)=1,\end{cases}
$$

where $2^{\alpha_{r}}\left\|2 r^{2}+k, 2^{\beta_{r}}\right\| r^{2}+k,\left(\alpha_{r} \geq 2, \beta_{r} \geq 2\right)$.

If

$$
(\bar{k}, K) \notin\{(0,-2),(1,-1),(2,-2),(3,-1)\},
$$

then the above relations with Lemma 3.1 imply that

$$
F(n)=\chi_{2 r^{2}}(n) \text { for every } n \in \mathbb{N}_{2 r^{2}} \text { and } r \in \mathcal{A},(r, 2)=1 .
$$

An application of Lemma 3.3 with $r=13$ and $r=15$ shows that

$$
F(n)=\chi_{\left(2 \cdot 13^{2}, 2 \cdot 15^{2}\right)}(n)=\chi_{2}(n)=1 \text { for every } n \in \mathbb{N}_{2},
$$

consequently

$$
F(2 n+1)=1 \text { for every } n \in \mathbb{N} .
$$

The assertion (3) of Theorem 1.2 is proved for the cases in (4.9).

Now we consider the following cases: $(\bar{k}, K) \in\{(0,-2),(1,-1),(2,-2),(3,-1)\}$.

It is clear to check that $8 n+3 \notin S$ for every $n \in \mathbb{N}_{0}$ and so Lemma 2.1 shows that $8 n+3=a^{2}+b^{2}+c^{2}$, $a, b, c \in \mathbb{Z}$. Since $a^{2}+b^{2}+c^{2} \equiv 3(\bmod 8)$, we have $a, b, c \in \mathbb{N}$ and $a \equiv b \equiv c \equiv 1(\bmod 2)$. Thus, we infer from (4.6) that

$$
F(8 n+3+k)=\chi_{2}(a)+\chi_{2}(b)+\chi_{2}(b)+K=3+K \neq 0 \text { if } K \in\{-1,-2\}
$$

for every $n \in \mathbb{N}$.

If $\bar{k}=1$, then $3+k=2^{\beta} t,(t, 2)=1, \beta \geq 2$ and by applying (4.10) with $n=2^{\beta-2} \ell$, we have

$$
F\left(2^{\beta}\right) F(2 \ell+t)=3+K \neq 0 \text { for every } \ell \in \mathbb{N} \text {. }
$$

This with Lemma 3.1 imply that

$$
F(n)=\chi_{2}(n)=1 \text { for every } n \in \mathbb{N}_{2},
$$

which proves (3) of Theorem 1.2.

If $\bar{k}=0$, then (4.8) and (4.10) with the facts $K \in\{-1,-2\}$ implies that

$$
\chi_{4}(3)=F(8 n+3+k)=3+K \in\{2,1\},
$$

which implies that $\chi_{4}(3)=1$. Thus, this relation with $(4.8)$ proves that $F(n)=\chi_{2}(n)=1$ for every $n \in \mathbb{N}_{2}$. 
Now we consider the case $(\bar{k}, K)=(2,-2), k=4 t+2, t \in \mathbb{N}_{0}$. Since $2 r \in \mathcal{A}$ for every $r \in \mathcal{A}$, therefore from (4.7) we obtain that

$$
\begin{aligned}
F(2) F\left(8 r^{2} n+2\left(r^{2}+t\right)+1\right) & =F\left(4(2 r)^{2} n+(2 r)^{2}+k\right) \\
& =\chi_{2}(2 r)+K=-2 \text { for every } n \in \mathbb{N}, r \in \mathcal{A} .
\end{aligned}
$$

We can find $r_{1}, r_{2} \in\{13,26\} \subset \mathcal{A}$ such that $r_{1}^{2}+t$ is odd and $r_{2}^{2}+t$ is even. Consequently, (4.8) and (4.11) show that

$$
F(2) F\left(8 r_{1}^{2} n+2\left(r_{1}^{2}+t\right)+1\right)=F(2) \chi_{4}(3)=-2
$$

and

$$
F(2) F\left(8 r_{2}^{2} n+2\left(r_{2}^{2}+t\right)+1\right)=F(2) \chi_{4}(1)=F(2)=-2 .
$$

These imply that $\chi_{4}(3)=1$, and so (4.8) implies that $F(n)=\chi_{2}(n)=1$ for every $n \in \mathbb{N}_{2}$. The assertion (3) of Theorem 1.2 is proved.

- Finally, we consider the case $(\bar{k}, K)=(3,-1)$. Let $k=4 q+3, q \in \mathbb{N}_{0}$ and $A, B, C \in \mathbb{N}$. Then, by setting $a=2 A, b=2 B$ and $c=2 C$ into (4.6), we infer from (4.8) that

$$
\begin{aligned}
\chi_{4}(3) & =\chi_{4}\left((2 A)^{2}+(2 B)^{2}+(2 C)^{2}+4 q+3\right)= \\
& =F\left((2 A)^{2}+(2 B)^{2}+(2 C)^{2}+4 q+3\right)= \\
& =\chi_{2}(2 A)+\chi_{2}(2 B)+\chi_{2}(2 C)-1=-1,
\end{aligned}
$$

which shows that

$$
F(2 n+1)=(-1)^{n} \text { for every } n \in \mathbb{N}
$$

Now we prove that

$$
F(2)=(-1)^{q+1} \cdot 2=(-1)^{\frac{k+1}{4}} \cdot 2 \text { and } F\left(2^{\alpha}\right)=0 \text { for every } \alpha \geq 2 .
$$

By setting $a=2 A+1, b=2 B+1$ and $c=2 C+1$ into (4.6), we infer from (4.12) that

$$
\begin{aligned}
F(2)(-1)^{q+1} & =F(2) F(4 t+2 q+3)=F(8 t+4 q+6)= \\
& =F\left((2 A+1)^{2}+(2 B+1)^{2}+(2 C+1)^{2}+4 q+3\right)= \\
& =\chi_{2}(2 A+1)+\chi_{2}(2 B+1)+\chi_{2}(2 C+1)-1=2,
\end{aligned}
$$

where $t=\frac{A(A+1)}{2}+\frac{B(B+1)}{2}+\frac{C(C+1)}{2}$. The last relation proves that $F(2)=(-1)^{q+1} \cdot 2$.

It can prove that if $\bar{k}=3$, then for each $\alpha \in \mathbb{N}, \alpha \geq 2$ there are $a, b, c \in \mathbb{N}$ such that $2|a, 2| b$, $(c, 2)=1$ and $2^{\alpha} \| a^{2}+b^{2}+c^{2}+k$. Therefore, (4.6) implies that

$$
F\left(2^{\alpha}\right) F\left(\frac{a^{2}+b^{2}+c^{2}+k}{2^{\alpha}}\right)=F\left(a^{2}+b^{2}+c^{2}+k\right)=\chi_{2}(a)+\chi_{2}(b)+\chi_{2}(c)-1=0,
$$

which completes the proof of (4.13). The proof of (4) is finished.

(3) Assume now that $(A, B, C)=(1,0,0)$ and $H(m)=m^{2}$ for every $m \in \mathbb{N}$. Then $D_{3}=3 C+K=K$ and

hold for every $\eta \in \mathbb{M}$, furthermore

$$
F(\eta+k)=\eta+K
$$

$$
F\left(4 r^{2} n+r^{2}+k\right)=4 r^{2} n+r^{2}+K \text { and } F\left(4 r^{2} n+2 r^{2}+k\right)=4 r^{2} n+2 r^{2}+K
$$

is satisfied for every $n \in \mathbb{N}$ and $r \in \mathcal{A}$. By using the assertion (c) of Lemma 3.2, we infer from (4.14) that

$$
k=K \text { and } F(n)=\chi_{4 r^{2}}(n) n \text { for every } n \in \mathbb{N}_{4 r^{2}} \text { and } r \in \mathcal{A} .
$$

On the other hand, it follows from (4.14) that

$$
\begin{cases}F(2) F\left(2 r^{2} n+r^{2}+\frac{k}{2}\right)=4 r^{2} n+2 r^{2}+k & \text { if } \bar{k}=0,(r, 2)=1, \\ F(2) F\left(2 r^{2} n+\frac{r^{2}+k}{2}\right)=4 r^{2} n+r^{2}+k & \text { if } \bar{k}=1,(r, 2)=1, \\ F\left(2^{\alpha_{r}}\right) F\left(2 r^{2} n+\frac{2 r^{2}+k}{2^{\alpha_{r}}}\right)=4 r^{2} n+2 r^{2}+k & \text { if } \bar{k}=2,(r, 2)=1, \\ F\left(2^{\beta_{r}}\right) F\left(2 r^{2} n+\frac{r^{2}+k}{2^{\beta_{r}}}\right)=4 r^{2} n+r^{2}+k & \text { if } \bar{k}=3,(r, 2)=1,\end{cases}
$$


where $2^{\alpha_{r}}\left\|2 r^{2}+k, 2^{\beta_{r}}\right\| r^{2}+k,\left(\alpha_{2} \geq 2, \beta_{r} \geq 2\right)$. These imply that

$$
F(n)=\chi_{2 r^{2}}(n) n \text { for every } n \in \mathbb{N}_{2 r^{2}} \text { and } r \in \mathcal{A} \text {. }
$$

We apply this relation for $r=13$ and $r=15$, Lemma 3.3 gives

$$
F(n)=\chi_{\left(2 \cdot 13^{2}, 2 \cdot 15^{2}\right)}(n) n=\chi_{2}(n) n \text { for every } n \in \mathbb{N}_{2},
$$

which implies that

$$
F(2 n+1)=2 n+1 \text { for every } n \in \mathbb{N} .
$$

Finally, we infer from Lemma 3.4 that for each $\alpha \in \mathcal{H}_{k}$ there is a $\eta \in \mathbb{M}$ such that $\eta+k=2^{\alpha} r,(r, 2)=1$. Thus, we have

$$
2^{\alpha} r=\eta+k=F(\eta+k)=F\left(2^{\alpha}\right) F(r)=F\left(2^{\alpha}\right) r
$$

and so $F\left(2^{\alpha}\right)=2^{\alpha}$.

The assertion (5) and Theorem 1.2 is proved.

\section{PROOFS OF COROLLARIES}

Proof of Corollary 1.3. Assume that $\ell \in \mathbb{N}, \ell \geq 3$ and the arithmetical functions $F: \mathbb{N} \rightarrow \mathbb{C}$ satisfy the relation

$$
F\left(n_{1}^{2}+\cdots+n_{\ell}^{2}\right)=F\left(n_{1}\right)^{2}+\cdots+F\left(n_{\ell}\right)^{2}
$$

holds for every $n_{1}, \ldots, n_{\ell} \in \mathbb{N}$.

From Theorem 1.1, there are numbers $A, B, C \in \mathbb{C}$ such that

$$
F(m)^{2}=A m^{2}+B \chi_{2}(m)+C
$$

and

$$
F\left(n_{1}^{2}+\cdots+n_{\ell}^{2}\right)=A\left(n_{1}^{2}+\cdots+n_{\ell}^{2}\right)+B\left(\chi_{2}\left(n_{1}\right)+\cdots+\chi_{2}\left(n_{k}\right)\right)+\ell C,
$$

hold for every $m, n_{1}, \ldots, n_{\ell} \in \mathbb{N}$. These imply that

$$
\begin{aligned}
& \left(A\left(n_{1}^{2}+\cdots+n_{\ell}^{2}\right)+B\left(\chi_{2}\left(n_{1}\right)+\cdots+\chi_{2}\left(n_{k}\right)\right)+\ell C\right)^{2}= \\
& =A\left(n_{1}^{2}+\cdots+n_{\ell}^{2}\right)^{2}+B \chi_{2}\left(n_{1}^{2}+\cdots+n_{\ell}^{2}\right)+C
\end{aligned}
$$

holds for every $n_{1}, \ldots, n_{\ell} \in \mathbb{N}$. This equation implies the following relations

$$
\left\{\begin{aligned}
A^{2} & =A \\
A\left(B\left(\chi_{2}\left(n_{1}\right)+\cdots+\chi_{2}\left(n_{k}\right)\right)+\ell C\right) & =0 \\
\left(B\left(\chi_{2}\left(n_{1}\right)+\cdots+\chi_{2}\left(n_{k}\right)\right)+\ell C\right)^{2} & =B \chi_{2}\left(n_{1}^{2}+\cdots+n_{\ell}^{2}\right)+C
\end{aligned}\right.
$$

hold for every $n_{1}, \ldots, n_{\ell} \in \mathbb{N}$. The solutions of this system are:

$$
(A, B, C) \in\left\{(0,0,0),\left(0,0, \frac{1}{\ell^{2}}\right),(1,0,0)\right\} \text {. }
$$

If $(A, B, C)=(0,0,0)$, then $F(n)=0$ for every $n \in \mathbb{N}$. If $(A, B, C)=\left(0,0, \frac{1}{\ell^{2}}\right)$, then $F(n)^{2}=\frac{1}{\ell^{2}}$, $F(n)=\frac{\varepsilon(n)}{\ell}$, where $\varepsilon(n) \in\{-1,1\}$ and $\varepsilon\left(n_{1}^{2}+\cdots+n_{\ell}^{2}\right)=1 .(A, B, C)=(1,0,0)$, then $F(n)^{2}=n^{2}$, $F(n)=\varepsilon(n) n$.

Thus, the proof of Corollary 1.3 is complete.

Proof of Corollary 1.4. Assume that $\ell \in \mathbb{N}, \ell \geq 4$ and the arithmetical functions $F: \mathbb{N} \rightarrow \mathbb{C}$ satisfy the relation

$$
F\left(n_{1}^{2}+\cdots+n_{\ell}^{2}\right)=F\left(n_{1}^{2}\right)+\cdots+F\left(n_{\ell}^{2}\right)
$$

holds for every $n_{1}, \ldots, n_{\ell} \in \mathbb{N}$. Similarly as the proof of Corollary 1.3 , there are numbers $A, B, C \in \mathbb{C}$ such that

$$
F\left(m^{2}\right)=A m^{2}+B \chi_{2}(m)+C
$$

holds for every $m \in \mathbb{N}$, and so

$$
F\left(n_{1}^{2}+\cdots+n_{\ell}^{2}\right)=A\left(n_{1}^{2}+\cdots+n_{\ell}^{2}\right)+B\left(\chi_{2}\left(n_{1}\right)+\cdots+\chi_{2}\left(n_{k}\right)\right)+\ell C .
$$


Since

$$
(2 \ell-3)^{2}=(2 \ell-4)^{2}+\underbrace{2^{2}+\cdots+2^{2}}_{l-2}+1^{2},
$$

we infer from (5.1) and (5.2) that

$$
F\left((2 \ell-3)^{2}\right)=A(2 \ell-3)^{2}+B \chi_{2}(2 \ell-3)+C
$$

and

$$
F\left((2 \ell-3)^{2}\right)=A(2 \ell-3)^{2}+B \chi_{2}(1)+\ell C .
$$

These imply that $\ell C=C$, consequently $C=0$. Finally, since $\ell \geq 3$, we have

$$
\begin{cases}\left(\frac{\ell}{2}\right)^{2}=\left(\frac{\ell-2}{2}\right)^{2}+\underbrace{1^{2}+\cdots+1^{2}}_{\ell-1} & \text { if } 2 \mid \ell \\ \left(\frac{\ell+3}{2}\right)^{2}=\left(\frac{\ell+1}{2}\right)^{2}+\underbrace{1^{2}+\cdots+1^{2}}_{\ell-2}+2^{2} & \text { if } 2 \nmid \ell,\end{cases}
$$

which with (5.1) and (5.2) imply

$$
\begin{cases}A\left(\frac{\ell}{2}\right)^{2}+B \chi_{2}\left(\frac{\ell}{2}\right)=A\left(\frac{\ell}{2}\right)^{2}+B\left(\chi_{2}\left(\frac{\ell-2}{2}\right)+\ell-1\right) & \text { if } 2 \mid \ell \\ A\left(\frac{\ell+3}{2}\right)^{2}+B \chi_{2}\left(\frac{\ell+3}{2}\right)=A\left(\frac{\ell+3}{2}\right)^{2}+B\left(\chi_{2}\left(\frac{\ell+1}{2}\right)+\ell-2\right) & \text { if } 2 \nmid \ell .\end{cases}
$$

One can check easily that these relations with $\ell \geq 4$ imply that $B=0$, consequently $F\left(m^{2}\right)=m^{2}$ for every $m \in \mathbb{N}$. Hence, from (5.2), we have

$$
F\left(n_{1}^{2}+\cdots+n_{\ell}^{2}\right)=A\left(n_{1}^{2}+\cdots+n_{\ell}^{2}\right)
$$

for every $n_{1}, \ldots, n_{\ell} \in \mathbb{N}$. The proof of Corollary 1.4 is finished.

\section{REFERENCES}

[1] ElLiotr, P. D. T. A. Probabilistic Number Theory I.. Grund. der Math. Wiss., 239, Springer-Verlag, New York, Berlin, 1979.

[2] Grosswald, E. Representations of Integers as Sums of Squares. Springer, 2011.

[3] Kнanн, B. M. M. On conjecture concerning the functional equation. Annales Univ. Sci. Budapest., Sect. Comp. 46 (2017), 123-135.

[4] Khanh, B. M. M. A note on a result of B. Bojan. Annales Univ. Sci. Budapest., Sect. Comp. 49 (2019), 285-297.

[5] Khanh, B. M. M. On the equation $f\left(n^{2}+D m^{2}+k\right)=f(n)^{2}+D f(m)^{2}+k$. Annales Univ. Sci. Budapest., Sect. Comp. 52 (2021), 217-241.

[6] PARK, Poo-Sung. Multiplicative function commutable with sums of squares. International Journal of Number Theory 14, 2 (2018), 469-478.

[7] PARK, Poo-SUng. On $k$-additive uniqueness of the set of squares for multiplicative functions. Aequationes mathematicae 92 (2018), 487-495.

[8] KátAi, I. And Phong, B. M. A characterization of functions using Lagrange’s Four-Square Theorem. Annales Univ. Sci. Budapest., Sect. Comp. 52 (2021), 177-185.

[9] Kátai, I. ANd Phong, B. M. Arithmetical functions commutable with sums of squares. Notes on Number Theory and Discrete Mathematics 27, 3 (2021), 143-154.

Open Access statement. This is an open-access article distributed under the terms of the Creative Commons Attribution 4.0 International License (https://creativecommons.org/licenses/by/4.0/), which permits unrestricted use, distribution, and reproduction in any medium, provided the original author and source are credited, a link to the CC License is provided, and changes - if any - are indicated. (SID_1) 